hep-th/0010155

AEI-2000-068

MPI-MIS-65/2000

\title{
An overview of new supersymmetric gauge theories with 2-form gauge potentials
}

\author{
Friedemann Brandt \\ Max-Planck-Institut für Mathematik in den Naturwissenschaften, \\ Inselstraße 22-26, D-04103 Leipzig, Germany \\ After October 1, 2000: \\ Max-Planck-Institut für Gravitationsphysik, Albert-Einstein-Institut, \\ Am Mühlenberg 1, D-14476 Golm, Germany
}

\begin{abstract}
An overview of new $4 \mathrm{~d}$ supersymmetric gauge theories with 2-form gauge potentials constructed by various authors during the past five years is given. The key rôle of three particular types of interaction vertices is emphasized. These vertices are used to develop a connecting perspective on the new models and to distinguish between them. One example is presented in detail to illustrate characteristic features of the models. A new result on couplings of 2 -form gauge potentials to Chern-Simons forms is presented.
\end{abstract}

\section{Introduction}

During the past five years, several new $4 \mathrm{~d}$ supersymmetric gauge theories have been constructed by various authors [1]-[13]. Common to all these models is the presence of 2 -form gauge potentials and a complicated (nonpolynomial) structure of interactions and symmetry transformations (gauge symmetries, supersymmetry). The initial motivation to construct such models came from string theory and focussed the attention first on the vector-tensor (VT) multiplet [14, 15] of $\mathrm{N}=2$ supersymmetry. Namely, in $\mathrm{N}=2$ supersymmetric $4 \mathrm{~d}$ heterotic string vacua, the dilaton is believed to reside in a VT multiplet (see, e.g., section 3 of the review [16]). In order to couple this multiplet to $\mathrm{N}=2$ supergravity, its so-called central charge must be gauged and this leads inevitably to the structures characteristic of the new models (cf. remarks at the end of section 3). Only two of the works [1]-13] are not devoted to the VT multiplet: in [1] a rather general class of new supersymmetric gauge theories with 2 -form gauge fields is constructed, and [13] deals with the double tensor (TT) multiplet of $\mathrm{N}=2$ supersymmetry and its couplings to vector and hyper multiplets. The TT multiplet is believed to be the dilaton-multiplet of $\mathrm{N}=2$ supersymmetric type IIB superstring vacua [16] and thus it should play there a rôle analogous to the VT multiplet in heterotic vacua. 
The purpose of this contribution is to give an overview of the new models and to emphasize the key rôle of three types of cubic interaction vertices in these models. To this end, first a brief excursion to consistent interactions of $p$-form gauge potentials in general is made in section 2. This will also show how the new models fit in the recent classification [17, 18, 19] of interactions between $p$-form gauge potentials. The three particular types of interaction vertices are identified and discussed in some detail in section 3, including a new result on couplings of 2-form gauge potentials to ChernSimons forms. Then these vertices and the supersymmetry multiplet structure are used to characterize the various models and to distinguish between them. In section 4 , an explicit example is treated in detail to illustrate characteristic features of the new models. The example is an $\mathrm{N}=2$ supersymmetric model found in 13], coupling the TT multiplet mentioned above to two $\mathrm{N}=2$ vector multiplets. Section 5 contains a selection of open problems and possible future developments.

\section{Interactions of $p$-form gauge potentials}

Gauge invariance restricts the possible interactions of $p$-form gauge fields quite severely. In the simplest case, the gauge transformation of a $p$-form gauge potential $A=$ $(1 / p !) d x^{\mu_{1}} \wedge \ldots \wedge d x^{\mu_{p}} A_{\mu_{1} \ldots \mu_{p}}$ is a natural generalization of the gauge transformation of the electromagnetic gauge field:

$$
\delta_{\text {gauge }}^{(0)} A=d \omega \Leftrightarrow \delta_{\text {gauge }}^{(0)} A_{\mu_{1} \ldots \mu_{p}}=p \partial_{\left[\mu_{1}\right.} \omega_{\left.\mu_{2} \ldots \mu_{p}\right]}
$$

where $\omega_{\mu_{1} \ldots \mu_{p-1}}$ are arbitrary gauge parameter fields. Analogously to the electromagnetic case, corresponding gauge invariant field strengths are thus

$$
F=d A \Leftrightarrow F_{\mu_{0} \ldots \mu_{p}}=(p+1) \partial_{\left[\mu_{0}\right.} A_{\left.\mu_{1} \ldots \mu_{p}\right]},
$$

and the standard Lagrangian for a set of free $p$-form gauge fields is a linear combination of Maxwell-type kinetic terms $F_{\mu_{0} \ldots \mu_{p}} F^{\mu_{0} \ldots \mu_{p}}$.

A systematic investigation of the possible interaction vertices which can be added consistently to such a free Lagrangian $L^{(0)}$ was carried out by Henneaux and Knaepen [17, 18, 19]. They studied consistent deformations of the free Lagrangian $L^{(0)}$ and of the gauge transformations $\delta_{\text {gauge }}^{(0)}$,

$$
\begin{aligned}
L & =L^{(0)}+g^{\alpha} V_{\alpha}^{(1)}+g^{\alpha} g^{\beta} V_{\alpha \beta}^{(2)}+\ldots \\
\delta_{\text {gauge }} & =\delta_{\text {gauge }}^{(0)}+g^{\alpha} \delta_{\text {gauge } \alpha}^{(1)}+g^{\alpha} g^{\beta} \delta_{\text {gauge } \alpha \beta}^{(2)}+\ldots
\end{aligned}
$$

where $g^{\alpha}$ are continuous coupling constants (deformation parameters), such that the deformed Lagrangian $L$ is invariant under the deformed gauge transformations $\delta_{\text {gauge }}$ modulo a total derivative,

$$
\delta_{\text {gauge }} L=\partial_{\mu} K^{\mu} \text {. }
$$

To first order in the coupling constants, (5) requires that the $V_{\alpha}^{(1)}$ be $\delta_{\text {gauge }}^{(0)}$-invariant on-shell in the free theory modulo a total derivative. Furthermore, without loss of 
generality, one may neglect all $V_{\alpha}^{(1)}$ which vanish on-shell in the free theory modulo a total derivative because they can be removed by field redefinitions (such vertices are therefore called trivial ones). Henneaux and Knaepen found the following result for the remaining first-order vertices:

Category 1: Vertices that are $\delta_{\text {gauge }}^{(0)}$-invariant off-shell modulo a total derivative and therefore do not modify the gauge transformations to first order. There are two types of such vertices (modulo total derivatives). Those of the first type depend on $p$-form gauge fields only via the field strengths $F_{\mu_{0} \ldots \mu_{p}}$ and their derivatives. Of course, there are infinitely many vertices of this type. Those of the second type are vertices of the Chern-Simons type

$$
A \wedge F \wedge \ldots \wedge F
$$

where the $F$ 's may have different form-degrees and all form-degrees must sum up to the spacetime dimension. These vertices are $\delta_{\text {gauge }}^{(0)}$-invariant only modulo a total derivative.

Category 2: Vertices that are $\delta_{\text {gauge }}^{(0)}$-invariant only on-shell in the free theory modulo a total derivative. These vertices are of particular interest because they are accompanied by deformations of the gauge transformations. A remarkable result is that, when ordinary gauge fields (1-form gauge potentials) are absent, all these vertices can be brought to the following form (modulo trivial vertices and vertices of category 1 ):

$$
A \wedge F \wedge \ldots \wedge F \wedge \underbrace{{ }^{*} F \wedge \ldots \wedge^{*} F}_{\text {at least one }{ }^{*} F}
$$

where ${ }^{*} F$ denotes the Hodge dual of $F$ and there must be at least one ${ }^{*} F$ because otherwise the vertex would be of the Chern-Simons type (6). Again, the F's may have different form-degrees and all form-degrees must sum up to the spacetime dimension. Therefore there are only finitely many vertices (7) for a finite number of $p$-form gauge fields. The first order deformations of the gauge transformations which correspond to a vertex $(\bar{\square})$ take the form

$$
\delta_{\text {gauge }}^{(1)} A=\omega \wedge F \wedge \ldots \wedge F \wedge^{*} F \wedge \ldots \wedge^{*} F
$$

where one of the ${ }^{*} F$ 's that occurs in (7) is omitted (for instance, when (7) contains only one ${ }^{*} F$, then (8) contains no ${ }^{*} F$ ). When 1 -form gauge potentials are present, (7) still gives nontrivial first-order vertices of category 2 , but then there may be additional vertices of category 2 which cannot be brought to the form (7). In particular, when at least three 1-form gauge potentials are present, there are Yang-Mills cubic vertices which differ from (7) because they contain two 'naked' gauge potentials instead of only one (the structure of Yang-Mills cubic vertices is $A \wedge A \wedge^{*} F$ where the $A$ 's are 1-form gauge potentials and $F$ is a 2-form field strength).

In four-dimensional spacetime there are three different types of cubic vertices (7) involving 1-form gauge potentials $A_{1}, 2$-form potentials $A_{2}$ and corresponding field strengths $F_{2}=d A_{1}$ and $F_{3}=d A_{2}$ :

$$
\begin{aligned}
& A_{2} \wedge^{*} F_{3} \wedge^{*} F_{3} \\
& A_{1} \wedge^{*} F_{2} \wedge^{*} F_{3} \\
& A_{1} \wedge F_{2} \wedge^{*} F_{3} .
\end{aligned}
$$

These are the vertices mentioned in the introduction. 


\section{Overview of the new models}

In accordance with commonly used nomenclature (which is actually somewhat unfair, see remarks at the end of this section), the vertices (9), (10) and (11) will be referred to as "Freedman-Townsend" (FT), "Henneaux-Knaepen" (HK) and "Chapline-Manton" $(\mathrm{CM})$ vertices, respectively. Each of the new supersymmetric models reviewed here contains at least one of these vertices. We label 1-form potentials and 2 -form potentials by indices $a=1,2, \ldots$ and $i=1,2, \ldots$ respectively, and denote their component fields by $A_{\mu}^{a}$ and $B_{\mu \nu}^{i}=-B_{\nu \mu}^{i}$. The field strengths of $A_{\mu}^{a}$ are denoted by $F_{\mu \nu}^{a}=\partial_{\mu} A_{\nu}^{a}-\partial_{\nu} A_{\mu}^{a}$, the Hodge-dualized field strengths of $B_{\mu \nu}^{i}$ by $H^{i \mu}=\frac{1}{2} \varepsilon^{\mu \nu \rho \sigma} \partial_{\nu} B_{\rho \sigma}^{i}$. The vertices (9), (10) and (11) read explicitly, using a suitable normalization,

$$
\begin{array}{cl}
\text { FT vertices: } & \frac{1}{4} f_{i j k} H_{\mu}^{i} H_{\nu}^{j} B_{\rho \sigma}^{k} \varepsilon^{\mu \nu \rho \sigma} \\
\text { HK vertices: } & T_{i a b} H_{\mu}^{i} F^{a \mu \nu} A_{\nu}^{b} \\
\text { CM vertices: } & \frac{1}{2} S_{i a b} H_{\mu}^{i} F_{\nu \rho}^{a} A_{\sigma}^{b} \varepsilon^{\mu \nu \rho \sigma}
\end{array}
$$

where the $f_{i j k}, T_{i a b}$ and $S_{i a b}$ are constant coefficients, with

$$
f_{i j k}=-f_{j i k} \quad, \quad S_{i a b}=S_{i b a} .
$$

$\left[S_{i a b}=S_{i b a}\right.$ can be imposed without loss of generality because $S_{i[a b]}$ can be removed from the vertices (14) by subtracting trivial vertices.] These coefficients are subject to conditions imposed by (5) at second order in the coupling constants (deformation parameters). Viewing $T_{i a b}$ and $S_{i a b}$ as the entries of matrices $T_{i}$ and $S_{i}$, these conditions read

$$
\begin{gathered}
f_{i j l} f_{k l m}+f_{j k l} f_{i l m}+f_{k i l} f_{j l m}=0 \\
{\left[T_{i}, T_{j}\right]=f_{i j k} T_{k}} \\
\left(S_{i} T_{j}-S_{j} T_{i}\right)+\left(S_{i} T_{j}-S_{j} T_{i}\right)^{\top}=f_{i j k} S_{k} .
\end{gathered}
$$

To derive these conditions, it was assumed that the zeroth order Lagrangian is $L^{(0)}=$ -(1/2) $H^{\mu i} H_{\mu}^{i}-(1 / 4) F_{\mu \nu}^{a} F^{\mu \nu a}$, and that (9), (10) and (11) are the only vertices of category 2 with non-vanishing coefficients (vertices of category 1 do not modify these conditions, but switching on other vertices of category 2 might cause modifications or lead to additional conditions).

(15) and (16) were already found in [17] and require that the $f_{i j k}$ be structure constants of a Lie algebra and that the $T_{i}$ be representation matrices of that Lie algebra, respectively. (17) was not derived in a previous work, to my knowledge. It requires that the symmetric parts of the matrices $2\left(S_{i} T_{j}-S_{j} T_{i}\right)$ be equal to $f_{i j k} S_{k}$. This is fulfilled, for instance, if $S_{i}=N T_{i}+T_{i}^{\top} N$ where $N$ is an arbitrary symmetric matrix (i.e., $S_{i a b}=N_{a c} T_{i c b}+N_{b c} T_{i c a}$ with $N_{a b}=N_{b a}$ ), but there are other solutions as well.

The corresponding first order deformations of the gauge transformations are

$$
\begin{aligned}
\delta_{\text {gauge }}^{(1)} B_{\mu \nu}^{i} & =-f_{i j k}\left(H_{\mu}^{j} \omega_{\nu}^{k}-H_{\nu}^{j} \omega_{\mu}^{k}\right)-\frac{1}{2} \varepsilon_{\mu \nu \rho \sigma} T_{i a b} F^{\rho \sigma a} \omega^{b}+S_{i a b} F_{\mu \nu}^{a} \omega^{b} \\
\delta_{\text {gauge }}^{(1)} A_{\mu}^{a} & =-T_{i a b} H_{\mu}^{i} \omega^{b} .
\end{aligned}
$$


The following table gives an overview of the new supersymmetric models. The vertices discussed above are used to distinguish between the various models. In addition the number of supersymmetries ( $\mathrm{N}=1$ or $\mathrm{N}=2$ supersymmetry) and the supersymmetry multiplets are given. In the case of $\mathrm{N}=1$ supersymmetry, $\mathrm{T}$ and $\mathrm{V}$ stand for tensor multiplets (also called linear multiplets) and vector multiplets respectively. In the case of $\mathrm{N}=2$ supersymmetry, VT, TT and V stand for vector-tensor multiplets, double-tensor multiplets and vector multiplets respectively.

\begin{tabular}{l|l|l|l} 
susy & multiplets & interactions & papers \\
\hline $\mathrm{N}=2$ & $\mathrm{VT}, \mathrm{V}$ & $\mathrm{HK}, \mathrm{CM}$ & [1, 2, [7, 8, 10 \\
\hline $\mathrm{N}=2$ & $\mathrm{VT}, \mathrm{V}$ & $\mathrm{CM}$ & [3, 4, 5, 9] \\
\hline $\mathrm{N}=2$ & $\mathrm{VT}$ & $\mathrm{CM}$ & {$[6]$} \\
\hline $\mathrm{N}=1$ & $\mathrm{~T}, \mathrm{~V}$ & $\mathrm{FT}, \mathrm{HK}, \mathrm{CM}$ & {$[1]$} \\
\hline $\mathrm{N}=2$ & $\mathrm{VT}$ & $\mathrm{HK}$ & {$[1]$} \\
\hline $\mathrm{N}=2$ & $\mathrm{TT}, \mathrm{V}$ & $\mathrm{FT}, \mathrm{HK}$ & 13
\end{tabular}

Of course, this table characterizes the various models only very roughly. The example in the next section is to illustrate characteristic features of these models. It is beyond the scope of this paper to review the various models in greater detail but I would like to add at least a few remarks: (a) Among all these models only those in [7] are locally supersymmetric, the other ones are globally supersymmetric. (b) The works on the VT multiplet overlap in part because some of these works rederive models which had already been found by means of other methods in previous works. (c) Models in the same row of the table may of course still differ. For instance, CM vertices in two models with the same multiplet content may contain different Chern-Simons forms (in the literature, this has led to a distinction between "linear" and "nonlinear" VT multiplets [2]). Different CM couplings correspond to different solutions to Eq. (17). Of course, analogous statements apply to the FT and HK vertices. (d) Some of the models in [11 possess extended $(N \geq 2)$ supersymmetry. For instance, it has been pointed out in 12 that the model constructed there can be obtained from [11]. However, it is not clear how to sieve out systematically those models in [11] which have extended supersymmetry.

Finally a few comments on the history may be in order. Models with FT interactions were constructed already by Ogievetsky and Polubarinov [20] a long time before the work by Freedman and Townsend [21]. CM interactions have a long history too. It seems that they appeared first in the early 80's [22, 23, 24] and, again, the work by Chapline and Manton was not the first one with such interactions. CM interactions attracted particular attention because of their crucial rôle in the Green-Schwarz anomaly cancellation mechanism [25] (the anomaly cancellation is made possible by the deformation of the gauge transformations associated with CM vertices, see section (2).

HK interactions (in four-dimensional spacetime) were discovered much later. However, the first models with such interactions were not found by Henneaux and Knaepen. Rather, it seems that HK interactions occurred for the first time in [1] where the central charge of the VT multiplet was gauged. The connection of that gauging to HK vertices is the following. Gauging the central charge (e.g., via the Noether method) gives rise to a vertex $V_{\mu} j^{\mu}$ where $V_{\mu}$ is a 1 -form gauge field and $j^{\mu}$ is the Noether current correspond- 
ing to the central charge symmetry. That Noether current is $j^{\mu}=H_{\nu} F^{\nu \mu}$, and thus the vertex $V_{\mu} j^{\mu}$ is a HK vertex. Combined FT and HK interactions, and the relation to Lie algebras, were found afterwards by Henneaux and Knaepen [17. It seems that the first and so far only work with models containing simultaneously FT, HK and CM vertices is [1].

\section{Example}

The example is an $\mathrm{N}=2$ supersymmetric model coupling one TT multiplet to two V multiplets and involves $\mathrm{HK}$ vertices but no FT or CM vertices. A TT multiplet contains two 2-form gauge potentials $B_{\mu \nu}^{i}(i=1,2)$, two real scalar fields $a^{i}$ and two Weyl fermions $\chi$ and $\psi$. Each $\mathrm{V}$ multiplet contains a 1 -form gauge potential $A_{\mu}$, a complex scalar field $\phi$ and two Weyl fermions $\lambda^{i}$. The $\mathrm{V}$ multiplets are labeled by the index $a=1,2$. This field content is supplemented with auxiliary fields $h_{\mu}^{i}$ which are embedded in the TT multiplet. These auxiliary fields allow one to construct the model in a compact polynomial form. In fact, it would be very cumbersome to construct the model without these auxiliary fields because of the complicated nonpolynomial structure which arises then, see below. Note that, in contrast to other supersymmetric models, the auxiliary fields do not lead to an off-shell closed supersymmetry algebra. On the contrary, the auxiliary fields make the supersymmetry algebra even "more open" (a formulation of the TT multiplet with an off-shell closed supersymmetry algebra is not known).

\begin{tabular}{c|cc|c} 
& \multicolumn{2}{|c|}{ bosons } & Weyl-fermions \\
\hline $\mathrm{TT}$ & $B_{\mu \nu}^{i} \quad a^{i} \quad\left(h_{\mu}^{i}\right)$ & $\chi \psi$ \\
\hline $\mathrm{V}^{a}$ & \multicolumn{2}{|c|}{$A_{\mu}^{a} \quad \phi^{a}$} & $\lambda^{a i}$
\end{tabular}

Thanks to the inclusion of the auxiliary fields, the Lagrangian takes the following simple form (using conventions as 26] adapted to the Minkowski metric $\operatorname{diag}(1,-1,-1,-1))$,

$$
\begin{array}{r}
L=\partial_{\mu} a^{i} \partial^{\mu} a^{i}+h_{\mu}^{i} h^{\mu i}+2 h_{\mu}^{i} H^{\mu i}-\mathrm{i} \chi \partial \bar{\chi}-\mathrm{i} \psi \partial \bar{\psi} \\
-\frac{1}{4} \hat{F}_{\mu \nu}^{a} \hat{F}^{a \mu \nu}+\frac{1}{2} \hat{D}_{\mu} \phi^{a} \hat{D}^{\mu} \bar{\phi}^{a}-2 \mathrm{i} \lambda^{i a} \hat{D} \bar{\lambda}^{i a}
\end{array}
$$

where

$$
\begin{aligned}
\hat{F}_{\mu \nu}^{a} & =\hat{D}_{\mu} A_{\nu}^{a}-\hat{D}_{\nu} A_{\mu}^{a}=\partial_{\mu} A_{\nu}^{a}+g^{i} h_{\mu}^{i} \varepsilon^{a b} A_{\nu}^{b}-(\mu \leftrightarrow \nu) \\
\hat{D}_{\mu} \phi^{a} & =\partial_{\mu} \phi^{a}+g^{i} h_{\mu}^{i} \varepsilon^{a b} \phi^{b} \\
\hat{D} \bar{\lambda}^{i a} & =\sigma^{\mu}\left(\partial_{\mu} \bar{\lambda}^{i a}+g^{i} h_{\mu}^{i} \varepsilon^{a b} \bar{\lambda}^{i b}\right) .
\end{aligned}
$$

The $g^{i}$ are real coupling constants (deformation parameters). Note that $\hat{D}_{\mu}$ has the form of a covariant derivative even though the auxiliary fields cannot be viewed as gauge fields (in fact, they substitute for field strengths, as the equations of motion give 
$\left.h_{\mu}^{i}=-H_{\mu}^{i}+\ldots\right)$. The auxiliary fields also simplify the structure of the gauge and supersymmetry transformations considerably. The gauge transformations read

$$
\begin{aligned}
\delta_{\text {gauge }} A_{\mu}^{a} & =\hat{D}_{\mu} \omega^{a}=\partial_{\mu} \omega^{a}+g^{i} h_{\mu}^{i} \varepsilon^{a b} \omega^{b} \\
\delta_{\text {gauge }} B_{\mu \nu}^{i} & =\frac{1}{4} g^{i} \omega^{a} \varepsilon^{a b} \varepsilon_{\mu \nu \rho \sigma} \hat{F}^{b \rho \sigma}+\partial_{\mu} \omega_{\nu}^{i}-\partial_{\nu} \omega_{\mu}^{i} \\
\delta_{\text {gauge }} & =0 \text { on other fields }
\end{aligned}
$$

where $\omega^{a}$ and $\omega_{\mu}^{i}$ are the gauge parameter fields associated with $A_{\mu}^{a}$ and $B_{\mu \nu}^{i}$ respectively. The supersymmetry transformations read, with constant anticommuting Weyl-spinors $\xi^{i}$ as transformation parameters,

$$
\begin{aligned}
\delta_{\text {susy }} A_{\mu}^{a}= & \varepsilon^{i j} \xi^{i} \sigma_{\mu} \bar{\lambda}^{j a}-\xi^{i} \Gamma^{i} \varepsilon^{a b} A_{\mu}^{b}+\text { c.c. } \\
\delta_{\text {susy }} \phi^{a}= & 2 \xi^{i} \lambda^{i a}-\left(\xi^{i} \Gamma^{i}+\bar{\xi}^{i} \bar{\Gamma}^{i}\right) \varepsilon^{a b} \phi^{b} \\
\delta_{\text {susy }} \lambda^{i a}= & \frac{\mathrm{i}}{2}\left(\varepsilon^{i j} \xi^{j} \sigma^{\mu \nu} \hat{F}_{\mu \nu}^{a}-\bar{\xi}^{i} \bar{\sigma}^{\mu} \hat{D}_{\mu} \phi^{a}\right)-\left(\xi^{j} \Gamma^{j}+\bar{\xi}^{j} \bar{\Gamma}^{j}\right) \varepsilon^{a b} \lambda^{i b} \\
\delta_{\text {susy }} B_{\mu \nu}^{i}= & -\varepsilon^{i j} \xi^{j} \sigma_{\mu \nu} \chi+\xi^{i} \sigma_{\mu \nu} \psi \\
& +\mathrm{i} g^{i} \varepsilon^{a b}\left(\bar{\phi}^{a} \xi^{j} \sigma_{\mu \nu} \lambda^{j b}+\varepsilon^{j k} A_{[\mu}^{a} \xi^{j} \sigma_{\nu]} \bar{\lambda}^{k b}\right)+\text { c.c. } \\
\delta_{\text {susy }} a^{i}= & \frac{1}{2}\left(\xi^{i} \chi-\varepsilon^{i j} \xi^{j} \psi\right)+\text { c.c. } \\
\delta_{\text {susy }} \chi= & -\bar{\xi}^{i} \bar{\sigma}^{\mu}\left(\varepsilon^{i j} h_{\mu}^{j}+\mathrm{i} \partial_{\mu} a^{i}\right) \\
\delta_{\text {susy }} \psi= & -\bar{\xi}^{i} \bar{\sigma}^{\mu}\left(h_{\mu}^{i}+\mathrm{i} \varepsilon^{i j} \partial_{\mu} a^{j}\right) \\
\delta_{\text {susy }} h_{\mu}^{i}= & \frac{\mathrm{i}}{2} \partial_{\mu}\left(\xi^{i} \psi-\varepsilon^{i j} \xi^{j} \chi\right)+\text { c.c. }
\end{aligned}
$$

where

$$
\Gamma^{i}=\frac{\mathrm{i}}{2} g^{j}\left(\varepsilon^{i j} \chi+\delta^{i j} \psi\right)
$$

The commutator algebra of the supersymmetry and gauge transformations is rather complicated off-shell but on-shell it is quite simple,

$$
\begin{aligned}
{\left[\delta_{\text {susy }}, \delta_{\text {susy }}^{\prime}\right] } & \approx \delta_{\text {translation }}+\delta_{\text {gauge }} \\
{\left[\delta_{\text {susy }}, \delta_{\text {gauge }}\right] } & \approx \delta_{\text {gauge }}^{\prime} \\
{\left[\delta_{\text {gauge }}, \delta_{\text {gauge }}^{\prime}\right] } & \approx 0
\end{aligned}
$$

where $\approx$ is equality on-shell. (20) is the standard $\mathrm{N}=2$ supersymmetry algebra onshell (modulo gauge transformations), with vanishing central charge. I remark that the gauge transformations which appear on the right hand side of (20) involve explicitly the spacetime coordinates, see [13] and [27] for details and comments on this point. (21) illustrates a feature typical of many of the new models, namely that gauge and supersymmetry transformations do not commute (not even on-shell). Explicitly, the gauge parameter fields $\omega^{a \prime}$ and $\omega_{\mu}^{i \prime}$ of $\delta_{\text {gauge }}^{\prime}$ on the right hand side of (21) read

$$
\begin{aligned}
\omega^{a \prime} & =\left(\xi^{i} \Gamma^{i}+\bar{\xi}^{i} \bar{\Gamma}^{i}\right) \varepsilon^{a b} \omega^{b} \\
\omega_{\mu}^{i \prime} & =-\frac{\mathrm{i}}{2} g^{i} \varepsilon^{a b} \varepsilon^{j k} \omega^{a}\left(\xi^{j} \sigma_{\mu} \bar{\lambda}^{k b}-\lambda^{k b} \sigma_{\mu} \bar{\xi}^{j}\right)
\end{aligned}
$$


where the $\xi$ 's and $\omega$ 's are supersymmetry parameters and gauge parameter fields of $\delta_{\text {susy }}$ and $\delta_{\text {gauge }}$ on the left hand side of (21). According to (22), the gauge transformations commute on-shell which is also typical of the new models [note: the algebra of the gauge transformations is not related to the Lie algebra underlying Eqs. (15) through (17)!].

Let me finally discuss the nonpolynomial structure which arises when one eliminates the auxiliary fields. The Lagrangian (19) contains the auxiliary fields at most quadratically,

$$
\begin{aligned}
L= & -\frac{1}{4} F_{\mu \nu}^{a} F^{a \mu \nu}+\partial_{\mu} a^{i} \partial^{\mu} a^{i}+\frac{1}{2} \partial_{\mu} \phi^{a} \partial^{\mu} \bar{\phi}^{a} \\
& -\mathrm{i} \chi \partial \bar{\chi}-\mathrm{i} \psi \partial \bar{\psi}-2 \mathrm{i} \lambda^{i a} \partial \bar{\lambda}^{i a}+2 h_{\mu}^{i} \mathcal{H}^{\mu i}+h_{\mu}^{i} K^{\mu i, \nu j} h_{\nu}^{j}
\end{aligned}
$$

where

$$
\begin{aligned}
& \mathcal{H}^{\mu i}=H^{\mu i}-g^{i} \varepsilon^{a b}\left(\frac{1}{2} F^{a \mu \nu} A_{\nu}^{b}+\frac{1}{4} \phi^{a} \stackrel{\leftrightarrow}{\partial^{\mu}} \bar{\phi}^{b}+\mathrm{i} \lambda^{j a} \sigma^{\mu} \bar{\lambda}^{j b}\right) \\
& K^{\mu i, \nu j}=\eta^{\mu \nu} \delta^{i j}+\frac{1}{2} g^{i} g^{j}\left[\eta^{\mu \nu}\left(\phi^{a} \bar{\phi}^{a}-A_{\rho}^{a} A^{a \rho}\right)+A^{a \mu} A^{a \nu}\right]
\end{aligned}
$$

The auxilary fields can be eliminated by solving their algebraic equations of motion. The solution is

$$
h_{\mu}^{i}=-\left(K^{-1}\right)_{\mu i, \nu j} \mathcal{H}^{\nu j}
$$

where $K^{-1}$ is the inverse of the field dependent matrix $K,\left(K^{-1}\right)_{\mu i, \rho k} K^{\rho k, \nu j}=\delta_{\mu}^{\nu} \delta_{i}^{j}$. Note that $K$ does not involve derivatives of the fields and therefore $K^{-1}$ is nonpolynomial in the fields but still local. Hence, using (23), the Lagrangian, gauge and supersymmetry transformations become nonpolynomial but remain strictly local. Expanding the resulting Lagrangian in the coupling constants, one finds at first order HK vertices as well as vertices of category 1 which complete the HK vertices such that the sum is supersymmetric on-shell in the free theory modulo a total derivative,

$$
\begin{aligned}
L= & -\frac{1}{4} F_{\mu \nu}^{a} F^{a \mu \nu}+\partial_{\mu} a^{i} \partial^{\mu} a^{i}+\frac{1}{2} \partial_{\mu} \phi^{a} \partial^{\mu} \bar{\phi}^{a} \\
& -\mathrm{i} \chi \partial \bar{\chi}-\mathrm{i} \psi \partial \bar{\psi}-2 \mathrm{i} \lambda^{i a} \partial \bar{\lambda}^{i a}-\mathcal{H}^{\mu i}\left(K^{-1}\right)_{\mu i, \nu j} \mathcal{H}^{\nu j} \\
= & L^{(0)}+\underbrace{g^{i} \varepsilon^{a b} H_{\mu}^{i} F^{a \mu \nu} A_{\nu}^{b}}_{\mathrm{HK} \text { vertices }}+\underbrace{g^{i} \varepsilon^{a b} H_{\mu}^{i}\left(\frac{1}{2} \phi^{a} \stackrel{\leftrightarrow}{\partial^{\mu}} \bar{\phi}^{b}+2 \mathrm{i} \lambda^{j a} \sigma^{\mu} \bar{\lambda}^{j b}\right)}_{\begin{array}{c}
\text { category 1 vertices } \\
\text { (susy completion of HK vertices) }
\end{array}}+\ldots
\end{aligned}
$$

It was mentioned already that nonpolynomial structures as in this example are typical of the new gauge theories. They cannot be avoided in models with FT or HK vertices because they are necessary consequences of these vertices, already in the nonsupersymmetric case. The use of appropriate auxiliary fields that simplify the construction is an almost indispensable tool for constructing complicated models of this type, especially supersymmetric ones. The finding of such auxiliary fields and their embedding in supersymmetry multiplets is in general a nontrivial and subtle ingredient of the construction. In contrast, models which contain CM vertices but no FT or HK vertices are simpler and the issue of auxiliary fields is less involved. In particular, such models are not necessarily nonpolynomial although supersymmetry often enforces a nonpolynomial dependence on scalar fields even in such models. 


\section{Comments}

The following is a selection of open problems which may point to possible further developments in the field:

(i) In my opinion, the rôle of the matter fields (scalar fields, fermions) in the new supersymmetric models has not been fully understood yet. In particular, the relation of scalar fields to the underlying geometry (Lie algebra) is somewhat mysterious. A better understanding of this issue might be a key to a deeper understanding of the supersymmetry structure of the models and to a more systematic construction of such models.

(ii) Systematic classifications of the possible consistent and supersymmetric interactions involving $p$-form gauge potentials, analogous to the classification [17, 18, 19] of non-supersymmetric interactions, are largely missing. An exception is the classification of the lowest dimensional interaction vertices involving a TT multiplet in [13]. Supersymmetry supplements (5) with the additional requirement $\delta_{\text {susy }} L=\partial_{\mu} M^{\mu}$ where $\delta_{\text {susy }}$ are the deformed supersymmetry transformations. This restricts the possible interactions as compared to the non-supersymmetric case, and relates coefficients of various interaction terms. A typical example is (24) where the coefficients of the HK vertices are related to coefficients of interaction vertices of category 1. In fact, supersymmetry can even completely forbid interactions which would be allowed if supersymmetry were not imposed. An example is the absence of $\mathrm{N}=2$ supersymmetric $\mathrm{CM}$ couplings of the TT multiplet [13]. Furthermore, it depends on the supersymmetry multiplet structure which interactions are possible. For instance, it was just mentioned that there are no $\mathrm{N}=2$ supersymmetric CM couplings involving the TT multiplet, whereas such couplings do exist for the VT multiplet (cf. table in section 3). Such results could be relevant in the context of string theory when comparing properties of different superstring vacua.

(iii) Locally supersymmetric models with FT or HK couplings are almost completely missing so far. In fact, the only exception is the work [7] where $\mathrm{N}=2$ supergravity models with VT multiplets were constructed. The construction of locally supersymmetric extensions of some of the other models could be of interest in the string theory context. In particular this applies to supergravity models with the TT multiplet because of the conjectured importance of this multiplet to type IIB superstring vacua (cf. introduction).

(iv) Recall that FT, HK and CM vertices are special cases of vertices (7). Nonsupersymmetric models in spacetime dimensions $>4$ with such vertices have been constructed already [17, 28]. Analogous globally or locally supersymmetric models in higher spacetime dimensions have not been constructed so far. In fact it seems that the only vertices (7) which have been used in supersymmetric models in spacetime dimensions $>4$ so far are the familiar CM vertices (14). For instance, these vertices occur in 10dimensional supergravity in connection with the Green-Schwarz anomaly cancellation mechanism (cf. remarks at the end of section 3). 


\section{References}

[1] P. Claus, B. de Wit, M. Faux, B. Kleijn, R. Siebelink and P. Termonia, The vectortensor supermultiplet with gauged central charge, Phys. Lett. B 373 (1996) 81-88, hep-th/9512143.

[2] P. Claus, B. de Wit, M. Faux and P. Termonia, Chern-Simons couplings and inequivalent vector-tensor multiplets, Nucl. Phys. B 491 (1997) 201-220, hepth/9612203.

[3] R. Grimm, M. Hasler and C. Herrmann, The N=2 vector-tensor multiplet, central charge superspace, and Chern-Simons couplings, Int. J. Mod. Phys. A 13 (1998) 1805-1816, hep-th/9706108.

[4] N. Dragon, S.M. Kuzenko and U. Theis, The vector-tensor multiplet in harmonic superspace, Eur. Phys. J. C 4 (1998) 717-721, hep-th/9706169.

[5] I. Buchbinder, A. Hindawi and B.A. Ovrut, A two-form formulation of the vectortensor multiplet in central charge superspace, Phys. Lett. B 413 (1997) 79-88, hepth/9706216.

[6] N. Dragon and S.M. Kuzenko, Self-interacting vector-tensor multiplet, Phys. Lett. B 420 (1998) 64-68, hep-th/9709088.

[7] P. Claus, B. de Wit, M. Faux, B. Kleijn, R. Siebelink and P. Termonia, N=2 supergravity Lagrangians with vector-tensor multiplets, Nucl. Phys. B 512 (1998) 148-178, hep-th/9710212.

[8] N. Dragon and U. Theis, Gauging the central charge, hep-th/9711025;

The linear vector-tensor multiplet with gauged central charge, Phys. Lett. B 446 (1999) 314-320, hep-th/9805199.

[9] E. Ivanov and E. Sokatchev, On non-linear superfield versions of the vector-tensor multiplet, Phys. Lett. B 429 (1998) 35-47, hep-th/9711038.

[10] N. Dragon, E. Ivanov, S.M. Kuzenko, E. Sokatchev and U. Theis, N=2 rigid supersymmetry with gauged central charge, Nucl. Phys. B 538 (1999) 411-450, hepth/9805152.

[11] F. Brandt and U. Theis, D=4, N=1 supersymmetric Henneaux-Knaepen models, Nucl. Phys. B 550 (1999) 495-510, hep-th/9811180.

[12] U. Theis, New N=2 supersymmetric vector-tensor interaction, Phys. Lett. B 486 (2000) 443-447, hep-th/0005044.

[13] F. Brandt, New N=2 supersymmetric gauge theories: the double tensor multiplet and its interactions, Nucl. Phys. B 587 (2000) 543-567, hep-th/0005086. 
[14] M.F. Sohnius, K.S. Stelle and P.C. West, Off-mass-shell formulation of extended supersymmetric gauge theories, Phys. Lett. B 92 (1980) 123-127.

[15] B. de Wit, V. Kaplunovsky, J. Louis and D. Lüst, Perturbative couplings of vector multiplets in N=2 heterotic string vacua, Nucl. Phys. B 451 (1995) 53-95, hepth/9504006.

[16] J. Louis and K. Förger, Holomorphic couplings in string theory, Nucl. Phys. Proc. Suppl. 55 B (1997) 33-64, hep-th/9611184.

[17] M. Henneaux and B. Knaepen, All consistent interactions for exterior form gauge fields, Phys. Rev. D 56 (1997) 6076-6080, hep-th/9706119.

[18] M. Henneaux and B. Knaepen, The Wess-Zumino consistency condition for p-form gauge theories, Nucl. Phys. B 548 (1999) 491-526, hep-th/9812140.

[19] M. Henneaux and B. Knaepen, A theorem on first-order interaction vertices for free p-form gauge fields, Int. J. Mod. Phys. A 15 (2000) 3535-3548, hep-th/9912052.

[20] V.I. Ogievetsky and I.V. Polubarinov, The notoph and its possible interactions, Yad. Fiz. 4 (1966) 216-223, Sov. J. Nucl. Phys. 4 (1967) 156-161.

[21] D.Z. Freedman and P.K. Townsend, Antisymmetric tensor gauge theories and nonlinear $\sigma$-models, Nucl. Phys. B 177 (1981) 282-296.

[22] H. Nicolai and P.K. Townsend, $N=3$ supersymmetry multiplets with vanishing trace anomaly: building blocks of the $N>3$ supergravities, Phys. Lett. B 98 (1981) 257-260.

[23] E. Bergshoeff, M. de Roo, B. de Wit and P. van Nieuwenhuizen, Ten-dimensional Maxwell-Einstein supergravity, its currents, and the issue of its auxiliary fields, Nucl. Phys. B 195 (1982) 97-136.

[24] G.F. Chapline and N.S. Manton, Unification of Yang-Mills theory and supergravity in ten dimensions, Phys. Lett. B 120 (1983) 105-109.

[25] M.B. Green and J.H. Schwarz, Anomaly cancellations in supersymmetric D=10 gauge theory and superstring theory, Phys. Lett. B 149 (1984) 117-122.

[26] J. Wess and J. Bagger, Supersymmetry and supergravity, Princeton Series in Physics (Princeton Univ. Press, Princeton 1992).

[27] F. Brandt, Hidden symmetries of supersymmetric p-form gauge theories, Phys. Lett. B (to appear), hep-th/0009133.

[28] F. Brandt, J. Simón and U. Theis, Exotic gauge theories from tensor calculus, Class. Quant. Grav. 17 (2000) 1627-1636, hep-th/9910177. 